* Cómo citar este artículo: Consani, N. (2020). Editorial. Relaciones Internacionales, 29(59), 103. https://doi.org/10.24215/23142766e103

"La historia de la humanidad es una larga marcha hacia la libertad"

Friedrich Hegel

"Solamente aquel que construye el futuro tiene derecho a juzgar el pasado"

Friedrich Nietzsche

\title{
LOS 30 AÑOS DEL IRI
}

En el discurso de cierre de nuestro X Congreso de Relaciones Internacionales que se desarrolló el pasado mes de noviembre, señalábamos todo lo realizado por el Instituto en sus 30 años.

Una mención especial nos merece las palabras de Ángel Plastino, expresidente de nuestra Universidad, que en el mismo acto nos decía: "que uno de sus orgullos en su gestión como Presidente de la UNLP fue haber participado en el creación del IRI..."

En una edición anterior de la Revista ( $\left.\mathrm{N}^{\circ} 39 / 2010\right)$ con motivo de otro aniversario, hicimos mención a la historia de nuestra institución en sus primeras dos décadas.

En esta editorial, mencionaremos las creaciones, las actividades, los proyectos concretados y los trabajos de los miembros del IRI durante estos últimos 10 años

- Se creó un nuevo Departamento EURASIA y el anterior Departamento de Malvinas, Antártida e Islas del Atlántico Sur, cambio su denominación hacia un enfoque más abarcativo como ATLANTICO SUR

- $\quad$ Se crearon nuevos CENTROS DE ESTUDIOS:

-Crimen Organizado Transnacional (CECOT) (2018), iniciativa de Carolina Sampó;

-Genero(s) y Relaciones Internacionales (CEGRI) (2018) Dulce Chaves;

-Italianos (2015) Juan Carlos Pérsico;

y se relanzaron:

-Canadienses (2018) Federico Borrone;

-Sudeste Asiático (CESEA) (2012) Ezequiel Ramoneda.

y nuevas CÁTEDRAS:

India (2010) 
Rusia (2011)

Azerbaiyán (2012)

Sahara Occidental (2012)

Unión Europea (2013)

México (2013)

Turquía (2014)

En 2015, nuestro DOCTORADO en RELACIONES INTERNACIONALES, fue categorizado "A" por la Comisión Nacional de Evaluación y Acreditación Universitaria (CONEAU)

En 2016, un grupo de jóvenes estudiantes universitarios de la provincia de Tucumán con la dirección de Patricia Patricia Kreibohm (Coordinadora del Departa-mento de Historia) generaron un nuevo espacio en el IRI, las EFEMÉRIDES, que se publican mensualmente.

En 2017, creamos una nueva Carrera de postgrado, la ESPECIALIZACION EN ESTUDIOS CHINOS, primera de este tipo en América Latina, uniendo Instituciones argentinas y chinas

Ese mismo año, se constituyó, por gestiones de Carolina Sampó (coordinadora del CECOT e integrante del Comité Doctoral) el Grupo JOVENES INVESTIGADORES del IRI

En 2018, el Departamento de Derecho Internacional, a través del proyecto de su Coordinadora Julia Espósito, editó el primer número de la REVISTA ELECTRONICA DE DERECHO INTERNACIONAL CONTEMPORANEO (2018)

En 2019, por iniciativa de Patricia Kreibohm y Jessica Petrino (Coordinadora y Secretaria del Departamento de Historia) fue instaurado el OBSERVATORIO UNIVERSITARIO DE TERRORISMO

Este año, dentro de nuestro Programa de Radio, se creó un nuevo Ciclo: las VOCES de TIEMPO INTERNACIONAL, con la conducción de nuestra Secretaria de Prensa: Silvina Fernández Cortés

Por otra parte, el Departamento de ASIA y el PACÍFICO continuó con la organización anual de la SEMANA DE ASIA y el PACÍFICO, y también se realizaron en forma bianual los ENCUENTROS CERPI-CENSUD y nuestro CONGRESO en RELACIONES INTERNACIONALES

Se continuaron editando los BOLETINES de:

- Centro de Reflexión en Política Internacional (CeRPI)

- América Latina y el Caribe

- Seguridad Internacional y Defensa

- Jóvenes Investigadores

- Derechos Humanos y Derecho Internacional Humanitario

- Estudios Canadienses 
- Asia y el Pacifico

Actualmente hay 3 Grupos de INVESTIGADORES y en la década hubo 8 proyectos terminados y aprobados

Se continuó con la enseñanza de los IDIOMAS:

- Chino Mandarín (a través del Instituto Confucio)

- Coreano

- Japonés

- Ruso (incorporado recientemente)

Se PUBLICARON, en el periodo:

- 22 Documentos de Trabajo

- 8 Libros

TESIS se aprobaron 30 de Maestría y 11 del Doctorado en RR.II.

REDES SOCIALES: EI IRI está presente en: Facebook - Instagram - Twitter - Youtube Finalmente, el Instituto cuenta desde 2012 con un ESPACIO DE ARTE

Como aporte ofrecemos:

ESTUDIOS (artículos sometidos a arbitraje)

En esta edición contamos con los trabajos de:

\section{Julieta Zelicovich}

Doctora en Relaciones Internacionales, Universidad Nacional de Rosario. Magíster en Relaciones Comerciales Internacionales, Universidad Nacional de Tres de Febrero. Investigadora Asistente en CONICET. Profesora de la licenciatura en Relaciones Internacionales de la Universidad Nacional de Rosario.

Email: julieta.zelicovich@fcpolit.unr.edu.ar

\section{Gerardo Caetano}

Doctor en Historia, Universidad Nacional de la Plata. Profesor Titular de la Universidad de la República. Coordinador del Observatorio de Coyuntura Política, Departamento de Ciencia Política. Investigador Nivel III del Sistema Nacional de Investigadores del Uruguay. Presidente del Consejo Superior de la Facultad Latinoamericana de Ciencias Sociales

\section{Nicolás Pose}

Candidato a Doctor en Ciencia Política, Universidad de la República. Magíster en Economía Política Internacional, London School of Economics and Political Science. Profesor asistente del Programa de Estudios Internacionales, Universidad de la República. Investigador Nivel Inicial del Sistema Nacional de Investigadores del Uruguay.

\section{Lucas Pavez Rosales}

Tercer Secretario del Servicio Exterior, Ministerio de Relaciones Exteriores de Chile. Magíster en Relaciones Internacionales de la Universidad Nacional de La Plata, Argentina. 
Profesor de Historia, Geografía y Ciencias Sociales, y Licenciado en Historia, mención Ciencia Política, de la Pontificia Universidad Católica de Valparaíso, Chile.

\section{Edwin Santiago Núñez Naranjo}

Magíster en Relaciones Internacionales, Universidad Andina Simón Bolívar, Doctorando del Programa de Desarrollo Regional e Integración Económica de la Universidad de Santiago de Compostela, Docente Tiempo Completo de la Universidad de Otavalo. Experto en negocios internacionales y Fundador de la empresa Strategyline, Quito- Ecuador.

\section{Mariana Colotta}

Licenciada en Sociología (USAL, 1995), Especialista en Metodología de la Investigación Científica (UNLA, 2004), Doctora en Ciencia Política (USAL, 2010). Doctora en Ciencias de la Educación (2018)

\section{Julio Ramón Lascano y Vedia}

Embajador del Servicio Exterior de la Nación. Licenciado en Ciencias Políticas y Relaciones Internacionales de la Universidad Católica Argentina (1983).

\section{Luciana Gil}

Doctora en Ciencias Sociales (Universidad de Buenos Aires), Investigadora asistente del Consejo Nacional de Investigaciones Científicas y Técnicas (CONICET), en el Instituto Interdisciplinario de Economía Política de la Facultad de Ciencias Económicas de la Universidad de Buenos Aires (IIEP-UBA). Docente en la UBA y en la Universidad de San Martín.

\section{Luís Antonio Paulino}

Profesor de la Universidade Estadual Paulista (UNESP) y Director del Instituto Confucio de la UNESP. Fue Asesor Especial del Ministerio de Hacienda (2003), Secretario Ejecutivo de la Secretaría de Coordinación Política y Asuntos Institucionales de la Presidencia de la República de Brasil (2003-2005) y Jefe de Asesoramiento del Ministerio de Deportes (20122014).

\section{Matías Bustamante Urzúa}

Administrador Público, Magíster en Ciencia Política, Universidad de Chile. Grupo de Investigación en Defensa, Fuerzas Armadas y Relaciones Internacionales, Instituto de Asuntos Públicos, Universidad de Chile.

\section{Sergio Cesarin}

Investigador del CONICET, Docente de la Especialización en Estudios Chinos del IRI, y Coordinador del Centro de Estudios sobre Asia del Pacífico e India (CEAPI) de la UNTREF

\section{Gabriel Balbo}

Docente-investigador de la Universidad Nacional Arturo Jaureche y UNLP, Coordinador del Programa Radar de Vigilancia Tecnológica e Inteligencia Competitiva VITC, UNAJ

En este número, nuevamente, publicamos un resumen de las últimas Tesis doctorales en Relaciones Internacionales defendidas y aprobadas: 


\section{Juan Camilo Mesa Bedoya}

Doctor en Relaciones Internacionales Universidad Nacional de La Plata, Internacionalista Universidad San Martín, Medellín, Colombia-Estado-empresas transnacionales: cambios en el régimen de solución de controversias inversor-Estado y redefinición de la soberanía a la luz de la protección de los derechos humanos

\section{Magdalena Bas Vilizzio}

Doctora en Relaciones Internacionales (Universidad Nacional de La Plata), Integrante del Sistema Nacional de Investigadores (Uruguay). E-mail: magdalena.bas@fder.edu.uy

\section{DIALOGOS}

Jorge Castro analista internacional. Fue Secretario de Planeamiento Estratégico de la Presidencia de la Nación (1998-99) Presidente del Instituto de Planeamiento

Estratégico (IPE).

\section{REFLEXIONES}

Ángel Tello Doctor en Relaciones Internacionales (IRI - UNLP).

Ex Secretario de Estrategia y Asuntos Militares (Ministerio de Defensa de la República Argentina), profesor de la Maestría y del Doctorado en Relaciones Internacionales del IRI UNLP

\section{HISTORIA}

Patricia Kreibohm, coordinadora del Departamento de Historia de las Relaciones Internacionales del IRI

Las restantes secciones se podrán consultar a través de la página Web del Instituto, www.iri.edu.ar, lo mismo que todos los números anteriores, desde la creación de la Revista, en diciembre de 1991.

Cronología,

Documentos,

Investigaciones,

Jurisprudencia,

Legislación,
Parlamentarias,

Política Exterior Argentina,

Publicaciones,

Tesis (Doctorado y Maestría en Relaciones Internacionales)

Hasta el próximo número

Prof. Dr. Norberto E. Consani

Director 
12 Relaciones Internacionales Vol 29 - № 59/2020. 